\title{
Pien Tze Huang inhibits tumor angiogenesis in a mouse model of colorectal cancer via suppression of multiple cellular pathways
}

\author{
ALING SHEN ${ }^{1,2^{*}}$, JIUMAO LIN $^{1,2^{*}}$, YOUQIN CHEN ${ }^{4 *}$, WEI LIN ${ }^{1}$, LIYA LIU $^{2}$, \\ ZHENFENG HONG ${ }^{1}$, THOMAS J. SFERRA ${ }^{4}$ and JUN PENG ${ }^{1,2,3}$ \\ ${ }^{1}$ Academy of Integrative Medicine and ${ }^{2}$ Fujian Key Laboratory of Integrative Medicine on Geriatrics, \\ Fujian University of Traditional Chinese Medicine, Minhou Shangjie, Fuzhou, Fujian 350122; \\ ${ }^{3}$ Postdoctor Workstation, Zhangzhou Pien Tze Huang Pharmaceutical Co., Ltd., Shangjie, \\ Zhangzhou, Fujian 363000, P.R. China; ${ }^{4}$ Rainbow Babies and Children's Hospital, \\ Case Western Reserve University School of Medicine, Cleveland, OH 44106, USA
}

Received May 22, 2013; Accepted June 24, 2013

DOI: 10.3892/or.2013.2609

\begin{abstract}
Angiogenesis plays an essential role in cancer progression, which therefore has become an attractive target for anticancer treatment. Tumor angiogenesis is tightly regulated by multiple signaling pathways that usually function redundantly; in addition, crosstalk between these pathways forms a complicated network that is regulated by compensatory mechanisms. Given the complexity of pathogenic mechanisms underlying tumor angiogenesis, most currently used angiogenesis inhibitors that only target single pathways may be insufficient and probably generate drug resistance, thus, increasing the necessity for development of novel anticancer agents. Traditional Chinese medicines (TCM) are receiving great interest since they have relatively fewer sideeffects and have been used for thousands of years to clinically treat various types of diseases including cancer. Pien Tze Huang (PZH), a well-known traditional Chinese formulation that was first prescribed 450 years ago, has long been used
\end{abstract}

Correspondence to: Dr Jun Peng, Academy of Integrative Medicine, Fujian University of Traditional Chinese Medicine, 1 Huatuo Road, Minhou Shangjie, Fuzhou, Fujian 350122, P.R. China

E-mail: pjunlab@hotmail.com

*Contributed equally

Abbreviations: PZH, Pien Tze Huang; TCM, traditional Chinese medicine; STAT3, signal transducer and activator of transcription 3; MAPK, mitogen-activated protein kinase; CRC, colorectal cancer; IHS, immunohistochemical staining; MVD, microvessel density; VEGF-A, vascular endothelial growth factor A; bFGF, basic fibroblast growth factor; NOS, nitric oxide synthase

Key words: Pien Tze Huang, traditional Chinese medicine, anticancer treatment, tumor angiogenesis, signaling pathway as an alternative remedy for cancers. However, the precise mechanism of PZH's anticancer activity remains to be further elucidated. Using a colorectal cancer mouse xenograft model, in the present study, we evaluated the effect of PZH on tumor angiogenesis and investigated the underlying molecular mechanisms. We found that PZH inhibited tumor growth since PZH treatment resulted in decrease in both tumor volume and tumor weight in CRC mice. In addition, PZH suppressed the activation of several signaling pathways such as STAT3, Akt and MAPKs. Consequently, the inhibitory effect of PZH on these pathways resulted in the inhibition of tumor angiogenesis as demonstrated by the decrease of microvessel density in tumor tissues. Moreover, PZH treatment reduced the expression of angiogenic factors including iNOS, eNOS, VEGF-A, bFGF as well as their specific receptors VEGFR2 and bFGFR. Altogether, our findings suggest that inhibition of tumor angiogenesis via suppression of multiple signaling pathways might be one of the mechanisms whereby PZH affects cancers.

\section{Introduction}

Angiogenesis, the formation of new blood vessels from the preexisting vasculatures, plays an important role in a wide range of biological processes including wound healing, reproduction and embryonic development. However, deregulation of this vital process is also essential for cancer progression (1-4). In the initial stage, tumor cells obtain oxygen and nutrients from nearby blood vessels by simple passive diffusion. However, when tumor grows to reach a size larger than $2 \mathrm{~mm}^{3}$, oxygen delivery by diffusion is no longer sufficient, which causes tumor cells to induce the sprouting of new blood vessels to support the continued growth of tumor and provide an avenue for hematogenous metastasis (5-9).

The process of angiogenesis is highly regulated by multiple cellular signaling transduction pathways including signal transducer and activator of transcription 3 (STAT3), serinethreonine kinase Akt and mitogen-activated protein kinases 
(MAPKs) (10-14). Aberrant activation of these pathways promotes tumor angiogenesis by inducing the expression of numerous critical angiogenic stimulators (15-17), including vascular endothelial growth factor A (VEGF-A), basic fibroblast growth factor (bFGF) and nitric oxide (NO) (18-22).

Due to the essential role of angiogenesis in cancer progression and metastasis, inhibition of tumor angiogenesis has become a promising strategy for anticancer chemotherapy. A variety of anti-angiogenic agents is currently in preclinical development, with some of them now entering clinical trials. However, the angiogenesis-related signaling pathways are usually redundant; and crosstalk between these pathways form a complicated and robust network that is regulated by compensatory mechanisms. Therefore, most currently used angiogenesis inhibitors that target only a single pathway may be insufficient and probably generate drug resistance (23). These problems highlight the need for the development of novel anticancer agents. Natural products, such as traditional Chinese medicine (TCM), have been used clinically to treat various kinds of diseases including cancer for thousands of years (24-26). TCM formula is a complex combination of many natural products, each of which contains numerous chemical compounds. TCM formulas, therefore, are considered to be multi-component and multi-target agents exerting their therapeutic function in a more holistic way. Pien Tze Huang (PZH) is a well-known traditional Chinese formulation that was first prescribed 450 years ago by a royal physician in the Ming Dynasty. The main ingredients of PZH include Moschus, Calculus Bovis, Snake Gall and Radix Notoginseng. These products together confer PZH properties of heatclearing, detoxification, promotion of blood circulation and removal of blood stasis (27). Since in the Chinese medicine system accumulation of toxic dampness and heat is one of the major causative factors in the pathogenesis of cancers, $\mathrm{PZH}$ is believed to be an effective anticancer agent. In fact, PZH has long been used as an alternative remedy for cancers in China and Southeast Asia. Recently, we reported that PZH can inhibit colorectal cancer growth in vivo and in vitro via promotion of cancer cell apoptosis and inhibition of cell proliferation, which is probably mediated by its inhibitory effect on activation of STAT3 pathway in tumor tissues (28-32). To further elucidate the mechanism of the tumoricidal activity of PZH, in the present we used a colorectal cancer mouse xenograft model to evaluate the effect of PZH on tumor angiogenesis and investigated the underlying molecular mechanisms.

\section{Materials and methods}

Materials and reagents. Pien Tze Huang (PZH) was obtained from and authenticated by the sole manufacturer Zhangzhou Pien Tze Huang Pharmaceutical Co., Ltd., China (Chinese FDA approval No: Z35020242). Dulbecco's modified Eagle's medium (DMEM), fetal bovine serum (FBS), penicillinstreptomycin, Trypsin-EDTA, TRIzol reagent, were purchased from Invitrogen (Grand Island, NY, USA). All antibodies were purchased from Santa Cruz Biotechnology (Santa Cruz, CA, USA). Bio-Plex phosphoprotein assay kits were purchased from Bio-Rad Laboratories (Hercules, CA, USA). All the other chemicals, unless otherwise stated, were obtained from Sigma Chemicals (St. Louis, MO, USA).
Cell culture. Human colon carcinoma HT-29 cells were obtained from the American Type Culture Collection (ATCC, Manassas, VA, USA). Cells were grown in DMEM containing $10 \%$ (v/v) FBS, 100 Units $/ \mathrm{ml}$ penicillin and $100 \mu \mathrm{g} / \mathrm{ml}$ streptomycin in a $37^{\circ} \mathrm{C}$ humidified incubator with $5 \% \mathrm{CO}_{2}$. The cells were subcultured at $80-90 \%$ confluency.

Animals. Athymic male nude mice were obtained from the Vital River Laboratory Animal Technology Co., Ltd. (Beijing, China) and housed in specific pathogen-free rooms in an environment with controlled temperature $\left(22^{\circ} \mathrm{C}\right)$, humidity, and a 12-h light/dark cycle. Food and water were given ad libitum throughout the experiment. All animal treatments were strictly in accordance with the international ethics guidelines and the National Institutes of Health Guide concerning the Care and Use of Laboratory Animals, and the experiments were approved by the Institutional Animal Care and Use Committee of the Fujian University of Traditional Chinese Medicine.

In vivo nude mice xenograft study. Cells $\left(1.5 \times 10^{6}\right)$ mixed with Matrigel (1:1) were subcutaneously injected in the right flank area of athymic nude mice to initiate tumor growth. After 3 days of xenograft implantation, mice were randomized into two groups $(\mathrm{n}=10)$ and given intragastric administration of $234 \mathrm{mg} / \mathrm{kg} / \mathrm{day}$ dose of PZH or saline daily, 5 days a week for 16 days. Tumor growth were measured every two days. Tumor growth was determined by measuring the major $(\mathrm{L})$ and minor (W) diameter with a caliper. The tumor volume was calculated according to the following formula: tumor volume $=\pi / 6 \mathrm{x} \mathrm{L} \mathrm{x}$ $\mathrm{W}^{2}$. At the end of the experiment, the animals were anaesthetized and the tumor tissue was removed and weighed.

Immunohistochemstry analysis. Tumor samples were fixed with $10 \%$ formaldehyde for $12 \mathrm{~h}$ and subsequently processed conventionally for paraffin-embedded tumor slides. The slides were subjected to antigen retrieval and the endogenous peroxidase activity was blocked with $3 \%$ hydrogen peroxide in water. For immunohistochemical staining, slides were incubated with rabbit polyclonal antibodies against CD31, VEGF-A, VEGFR2, bFGF, bFGFR, iNOS or eNOS (all in 1:200 dilution; Santa Cruz Biotechnology). After washing with PBS, slides were incubated with biotinylated secondary antibody followed by conjugated horseradish peroxidase (HRP)-labelled streptavidin (Dako) and then washed with PBS. The slides were then incubated with diamino-benzidine (DAB, Sigma) as the chromogen, followed by counterstaining with diluted Harris hematoxylin (Sigma). After staining, five high-power fields (x400) were randomly selected in each slide, and the average proportion of positive cells in each field were counted using the true color multi-functional cell image analysis management system (Image-Pro Plus; Media Cybernetics, Silver Spring, MD, USA). To rule out any non-specific staining, PBS was used to replace the primary antibody as a negative control.

Bio-Plex phosphoprotein assay. Tumors were homogenized and then lysed using a commercially available lysis kit (BioRad Laboratories), followed by centrifugation at $14,000 \mathrm{x} g$ for $15 \mathrm{~min}$. Protein concentrations of the clarified supernatants were determined by BCA protein assay. The presence of p-STAT3, p-Akt, p-Erk1/2, p-JNK and p-p38 was detected 

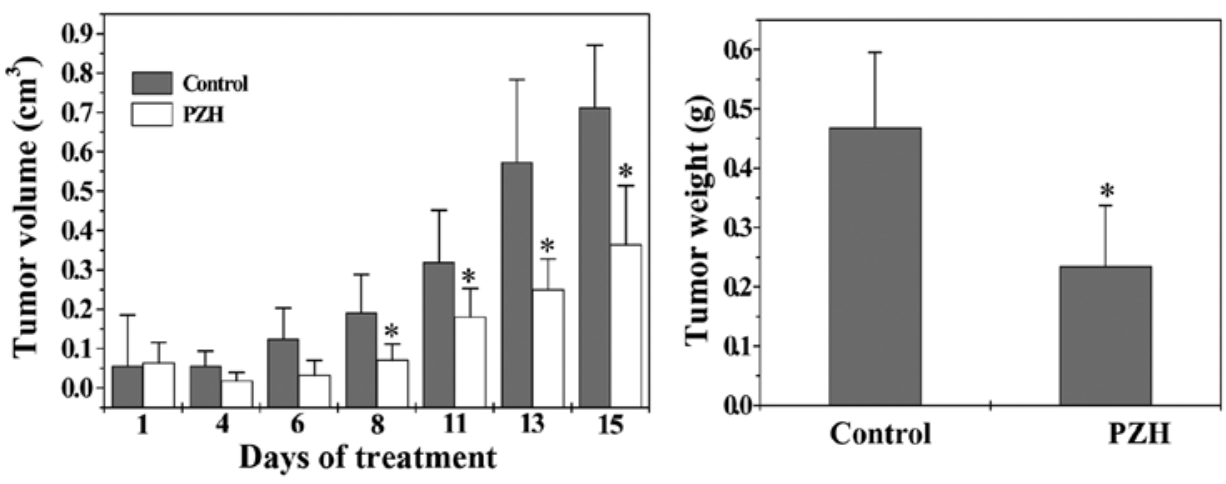

Figure 1. Effect of PZH on tumor growth in CRC xenograft mice. After tumor development, the mice were given intragastric administration of $234 \mathrm{mg} / \mathrm{kg}$ of PZH or saline daily, 5 days a week for 15 days. Tumor volume and tumor weight were measured. Data shown are the averages with SD (error bars) from 10 mice in each group. ${ }^{*} \mathrm{P}<0.05$, vs. controls
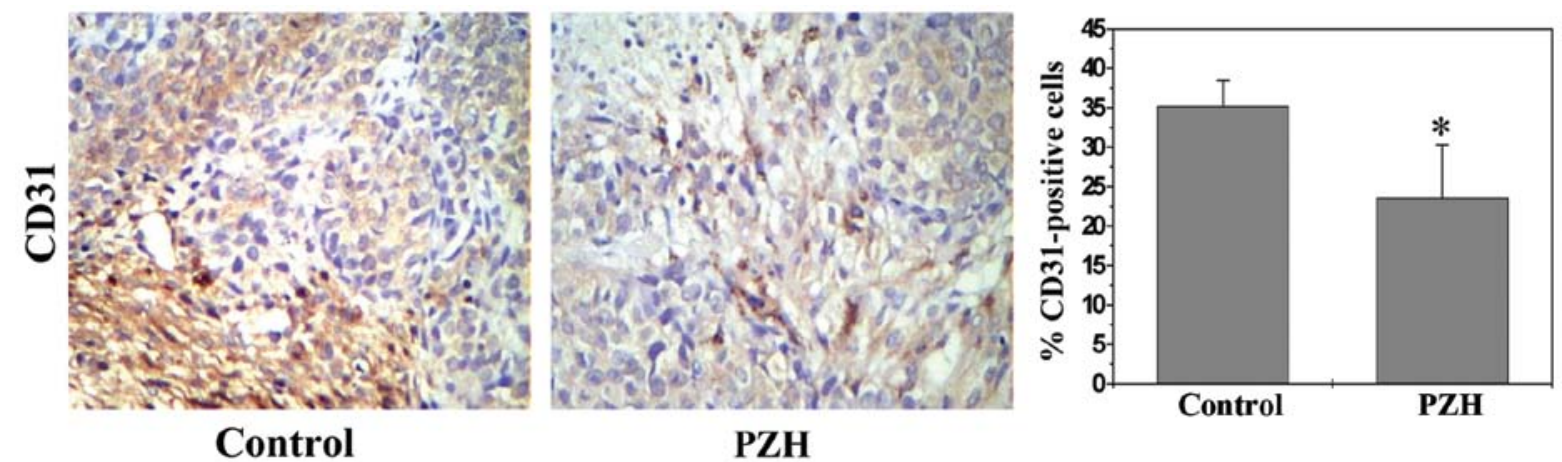

Figure 2. Effect of PZH on microvessel density (MVD) in CRC xenograft mice. At the end of the study, tumor tissues were processed for IHS for CD31. The photographs are representative images taken at a magnification of x 400 . Quantification of IHS assay is presented as percentage of positively-stained cells. Data shown are the average with $\mathrm{SD}$ (error bars) from 10 mice in each group. ${ }^{*} \mathrm{P}<0.05$, vs. controls.

using a bead-based multiplex assay for phosphoproteins (Bio-Plex phosphoprotein assay; Bio-Rad Laboratories) according to the manufacturer's protocol. Data were collected and analyzed using the Bio-Plex 200 suspension array system (Bio-Rad Laboratories).

Statistical analysis. Data are presented as mean \pm SD for the indicated number of independently performed experiments. Statistical analysis was carried out with the Student's t-test. Differences with $\mathrm{P}<0.05$ were considered to be statistically significant.

\section{Results and Discussion}

PZH suppresses colorectal cancer growth via inhibition of tumor angiogenesis. The therapeutic efficacy of $\mathrm{PZH}$ against tumor growth was evaluated through comparison of tumor weight and volume in treated and control CRC xenograft mice. As shown in Fig. 1, tumor growth was significantly suppressed by PZH treatment throughout the experiment. The final tumor volume or tumor weight per mouse in control group was $0.71 \pm 0.16 \mathrm{~cm}^{3}$ or $0.47 \pm 0.13 \mathrm{~g}$; while that in PZH-treated group was $0.36 \pm 0.15 \mathrm{~cm}^{3}$ or $0.23 \pm 0.10 \mathrm{~g}(\mathrm{P}<0.05)$, demonstrating that PZH is effective in suppressing colorectal tumor growth.

Angiogenesis plays an essential role in cancer development; we therefore investigated the effect of PZH on intratumoral microvessel density (MVD) that is an indicator of new blood vessel growth. Tumors from CRC xenograft mice were evaluated by immunohistochemical staining (IHS) for the expression of an endothelial cell-specific marker CD31; and data in Fig. 2 show that the percentage of CD31-positive cells in control or PZH-treated mice was $35.22 \pm 3.26$ or $23.60 \pm 6.73 \%$, respectively $(\mathrm{P}<0.05)$, suggesting that $\mathrm{PZH}$-caused inhibition of tumor growth is associated with its anti-angiogenic activity.

PZH inhibits the expression of VEGF-A, VEGFR2, bFGF and $b F G F R$. As most potent angiogenic stimulators, VEGF-A and bFGF are commonly overexpressed in many kinds of human cancer, which is correlated with tumor progression, invasion and metastasis, and poorer survival and prognosis in patients (33-36). VEGF-A and bFGF exert their biological function primarily through interaction with their specific receptors located on the surface of vascular endothelial cells, such as VEGFR-2 and bFGFR. Binding of VEGF-A and bFGF and their receptors leads to receptor dimerization, which in turn activates downstream signaling cascades such as the Akt and Erk pathways, leading to the proliferation, migration, survival, sprouting and eventually tube formation of endothelial cells (35-37).

By performing IHS analysis we found that PZH treatment profoundly inhibited the expression of VEGF-A and bFGF as well as their receptors in tumor tissues. The percentage of VEGF-A, VEGFR2, bFGF or bFGFR-positive cells in control group was, respectively, $38.77 \pm 7.76,31.38 \pm 3.36,23.80 \pm 6.73$ 

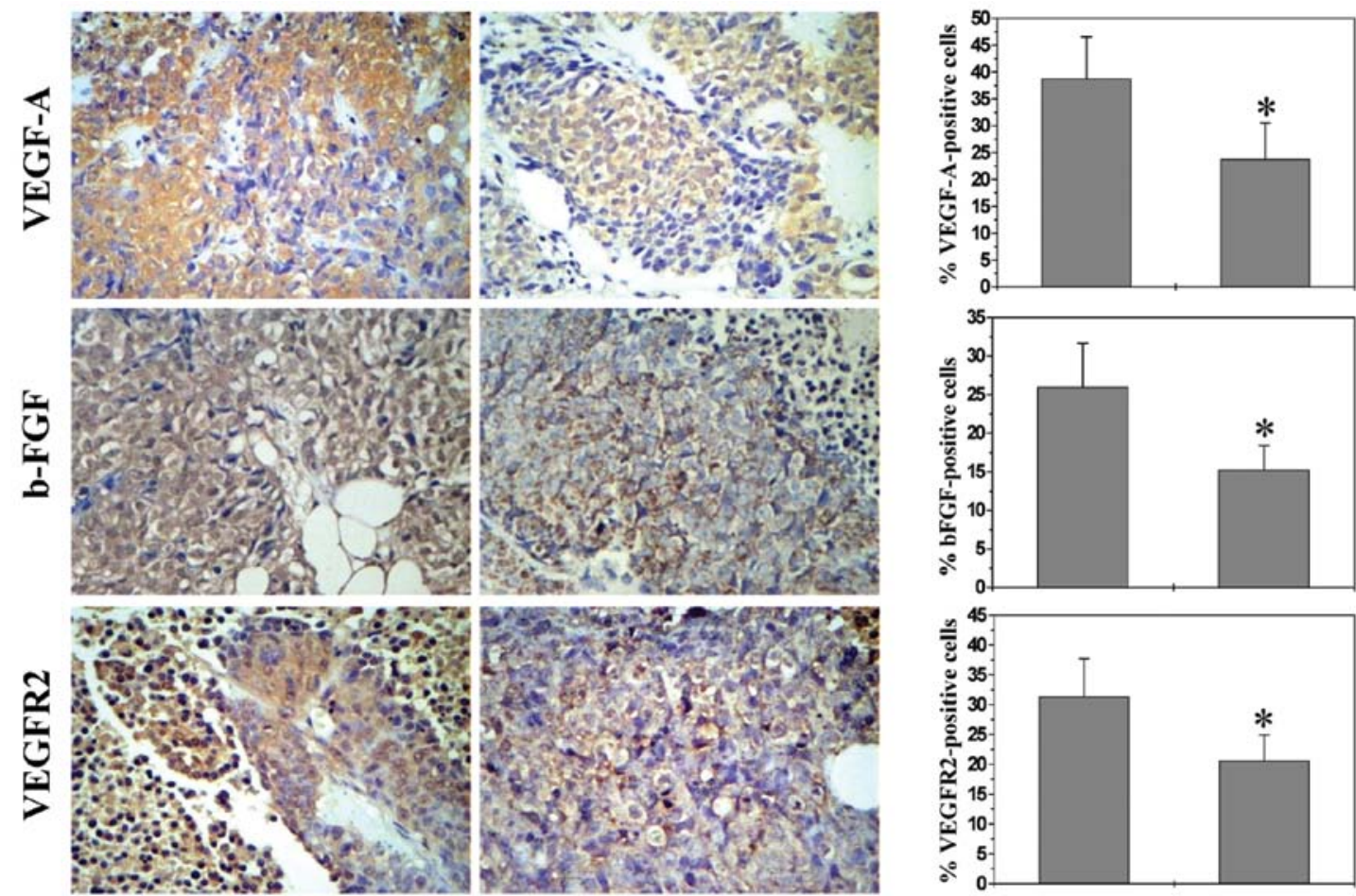

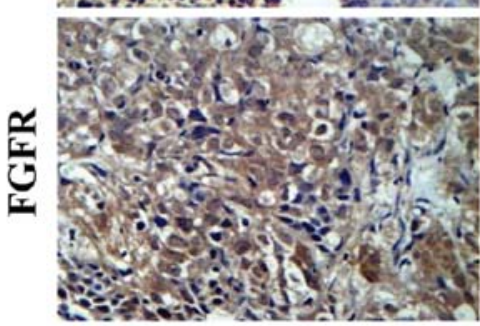

Control

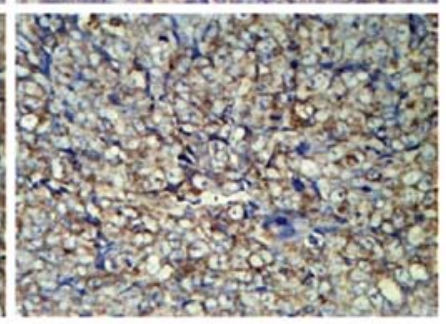

PZH
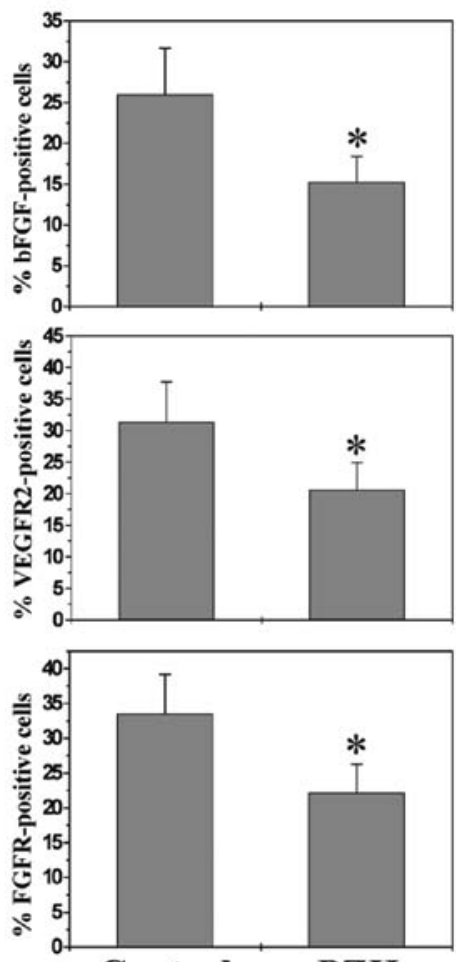

Control PZH

Figure 3. Effect of PZH on the expression of VEGF-A, VEGFR2, bFGF and bFGFR in CRC xenograft mice. Tumor tissues were processed for IHS for VEGF-A, VEGFR2, bFGF and bFGFR. The photographs are representative images taken at a magnification of x400. Quantification of IHS assay is presented as percentage of positively-stained cells. Data shown are the average with SD (error bars) from 10 mice in each group. ${ }^{*} \mathrm{P}<0.05$, vs. controls.
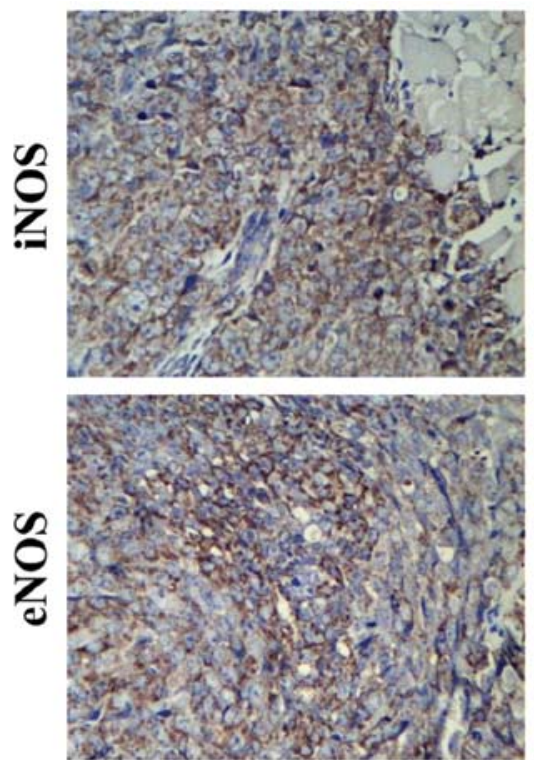

Control
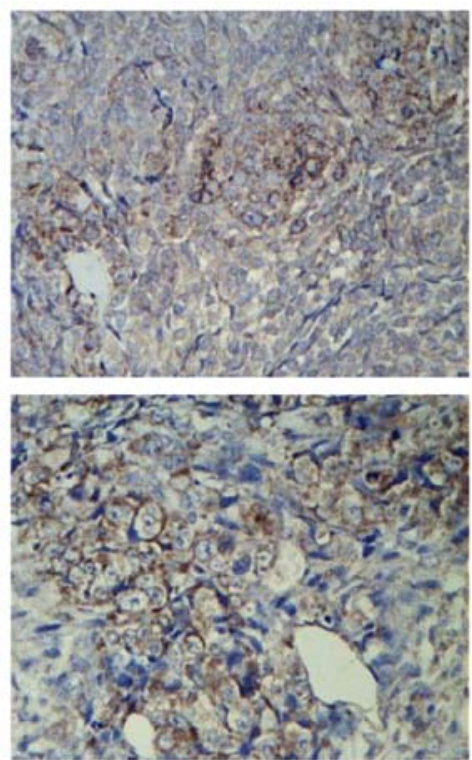

PZH
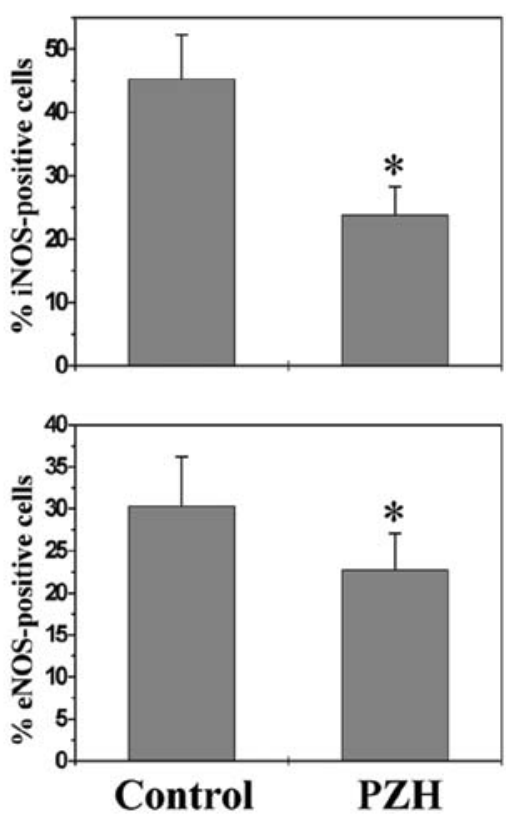

Figure 4. Effect of PZH on the expression of iNOS and eNOS in CRC xenograft mice. Tumor tissues were processed for IHS for iNOS and eNOS. The photographs are representative images taken at a magnification of x400. Quantification of IHS assay is presented as percentage of positively-stained cells. Data shown are the average with SD (error bars) from 10 mice in each group. ${ }^{*} \mathrm{P}<0.05$, vs. controls. 

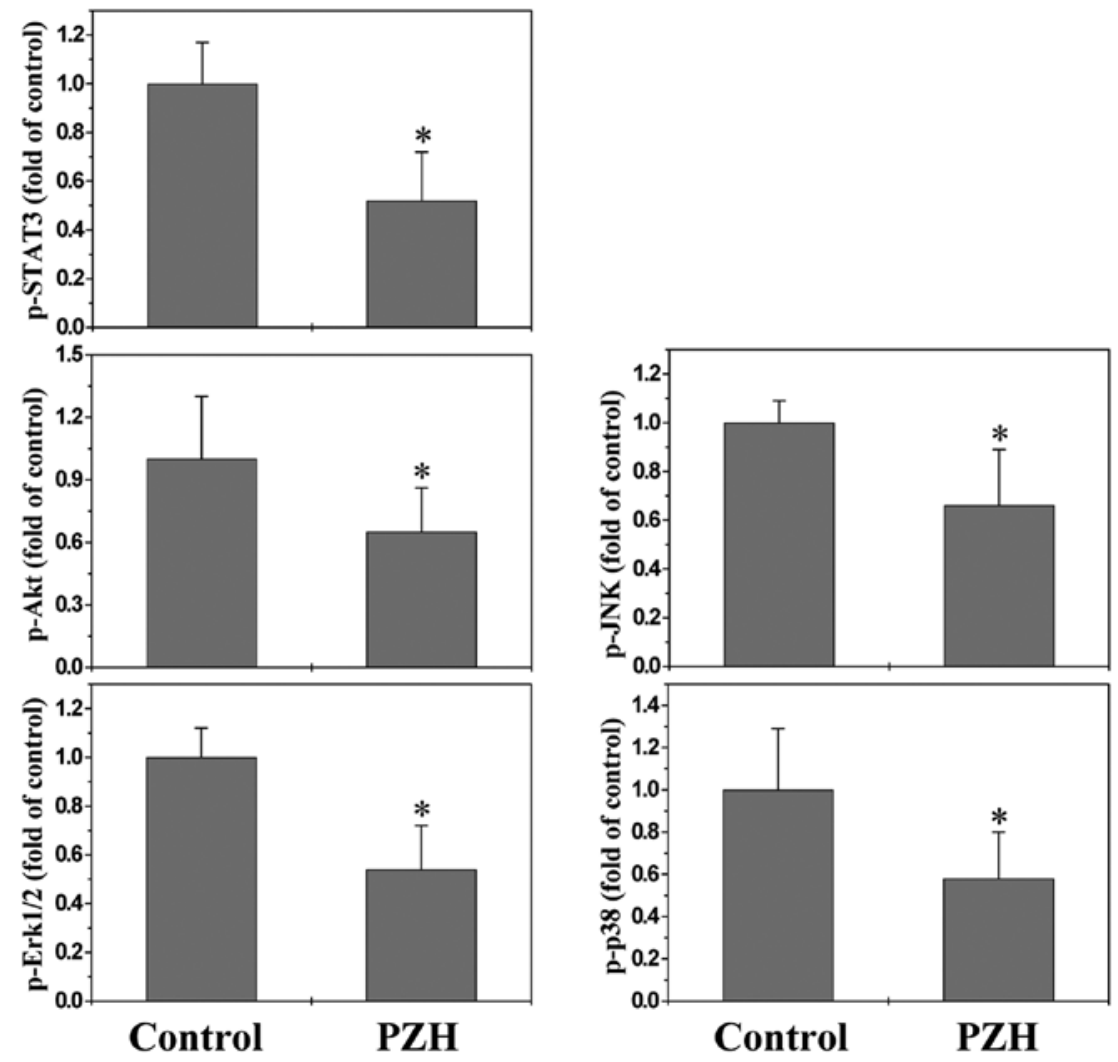

Figure 5. Effect of PZH on phosphorylation of STAT3, Akt, Erk1/2, JNK and p38 in CRC xenograft mice. The phosphorylation levels of STAT3, Akt, Erk1/2, JNK and p38 in tumor tissues were determined by Bio-Plex phosphoprotein assay. The data are normalized to the phosphorylation level within controls and presented as fold of control. Data are the average with SD (error bars) from 10 mice in each group. " $\mathrm{P}<0.05$, vs. controls.

and $33.50 \pm 5.67 \%$, whereas that in PZH-treated mice was 26.0 $\pm 5.67,20.60 \pm 4.32,15.25 \pm 3.17$ and $22.20 \pm 4.07 \%$ (Fig. 3; $\mathrm{P}<0.05)$.

PZH inhibits expression of iNOS and eNOS. Nitric oxide synthases (NOSs), particularly the inducible (iNOS) and endothelial (eNOS) isoforms, have been strongly implicated in tumor angiogenesis $(21,22,37-41)$; we therefore determined the effect of PZH on NOS expression. As shown in Fig. 4, the percentage of iNOS- or eNOS-positive cells in control group was, respectively, $45.33 \pm 6.93$ and $30.33 \pm 5.87 \%$, while that in PZH-treated mice was $23.80 \pm 4.52$ and $22.75 \pm 4.34 \%$ $(\mathrm{P}<0.05)$, suggesting that iNOS and eNOS could be potential molecular targets for the antitumor and anti-angiogenic effects of PZH.

PZH suppresses the activation of multiple signaling pathways. Cancer development is tightly regulated by multiple intracellular signaling pathways, including STAT3, Akt, ERK, JNK and p38. Aberrant activation of these pathways alters the expression of various critical target genes, such as above-mentioned angiogenic factors and NOSs, leading to the promotion of tumor angiogenesis. To further elucidate the underlying mechanisms of antitumor activity of $\mathrm{PZH}$, we determined its effect on the activation of STAT3, Akt, ERK, JNK and p38 pathways. The activation (phosphorylation) of STAT3, Akt, Erk1/2, JNK and p38 in xenograft tumor tissues was determined by Bio-Plex phosphoprotein assay. As shown in Fig. 5, after PZH treatment the phosphorylation levels of STAT3, ERK, Akt, JNK and p38 in tumors were decreased as compared to controls $(\mathrm{P}<0.05)$, suggesting that $\mathrm{PZH}$ exerts its antitumor activity probably through affecting multiple intracellular targets.

In conclusion, in the present study, we demonstrate for the first time that PZH inhibits colorectal cancer growth via suppression of multiple intracellular signaling pathways leading to the inhibition of tumor angiogenesis, which may in part explain its anticancer activity.

\section{Acknowledgements}

The present study was sponsored by the National Natural Science Foundations of China (81073097 and 81202790), the Developmental Fund of Chen Keji Integrative Medicine (CKJ 2011001) and the China Postdoctoral Science Foundation (2012M511437).

\section{References}

1. Folkman J: Tumor angiogenesis: therapeutic implications. $\mathrm{N}$ Engl J Med 285: 1182-1186, 1971.

2. Folkman J and Shing Y: Angiogenesis. J Biol Chem 267: 10931-10934, 1992.

3. Folkman J: Angiogenesis in cancer, vascular, rheumatoid and other diseases. Nat Med 1: 27-31, 1995.

4. Folkman J: Angiogenesis. Annu Rev Med 57: 1-18, 2006

5. Cook KM and Figg WD: Angiogenesis inhibitors: current strategies and future prospects. CA Cancer J Clin 60: 222-243, 2010. 
6. Mantovani A, Allavena P, Sica A and Balkwill F: Cancer related inflammation. Nature 454: 436-444, 2008.

7. Whiteside TL: The tumor microenvironment and its role in promoting tumor growth. Oncogene 27: 5904-5912, 2008.

8. Jain RK: Transport of molecules in the tumor interstitium: a review. Cancer Res 47: 3039-3051, 1987.

9. Folkman J: How is blood vessel growth regulated in normal and neoplastic tissue? G.H.A. Clowes memorial award lecture. Cancer Res 46: 467-473, 1986.

10. Robert S and Kerbel RS: Tumor angiogenesis. N Engl J Med 358: 2039-2049, 2008.

11. Sun W: Angiogenesis in metastatic colorectal cancer and the benefits of targeted therapy. J Hematol Oncol 5: 63, 2012.

12. Weis SM and Cheresh DA: Tumor angiogenesis: molecular pathways and therapeutic targets. Nat Med 17: 1359-1370, 2011.

13. Qian WF, Guan WX, Gao Y, Tan JF, Qiao ZM, Huang H and Xia CL: Inhibition of STAT3 by RNA interference suppresses angiogenesis in colorectal carcinoma. Braz J Med Biol Res 44: $1222-1230,2011$

14. Berra E, Pagès G and Pouysségur J: MAP kinases and hypoxia in the control of VEGF expression. Cancer Metastasis Rev 19: $139-145,2000$.

15. Stromblad S and Cheresh DA: Integrins, angiogenesis and vascular cell survival. Chem Biol 3: 881-885, 1996.

16. Breier $\mathrm{G}$ and Risau W: The role of vascular endothelial growth factor in blood vessel formation. Trends Cell Biol 6: 454-456, 1996.

17. Weidner N, Semple JP, Welch WR and Folkman J: Tumor angiogenesis and metastasis-correlation in invasive breast carcinoma. N Engl J Med 324: 1-8, 1991.

18. Ferrara N: Role of vascular endothelial growth factor in physiologic and pathologic angiogenesis: therapeutic implications. Semin Oncol 29: 10-14, 2002.

19. Jain RK: Tumor angiogenesis and accessibility: role of vascular endothelial growth factor. Semin Oncol 29: 3-9, 2002.

20. Risau W: Mechanisms of angiogenesis. Nature 386: 671-674, 1997.

21. Ziche $\mathrm{M}$ and Morbidelli L: Molecular regulation of tumour angiogenesis by nitric oxide. Eur Cytokine Netw 20: 164-170, 2009.

22. Cooke JP and Losordo DW: Nitric oxide and angiogenesis. Circulation 105: 2133-2135, 2002.

23. Eikesdal HP and Kalluri R: Drug resistance associated with antiangiogenesis therapy. Semin Cancer Biol 19: 310-317, 2009.

24. Newman DJ, Cragg GM and Snader KM: The influence of natural products upon drug discovery. Nat Prod Rep 17: 215-234, 2000.

25. Gordaliza M: Natural products as leads to anticancer drugs. Clin Transl Oncol 9: 767-776, 2007.

26. Ji HF, Li XJ and Zhang HY: Natural products and drug discovery. EMBO Rep 10: 194-200, 2009.

27. Chinese Pharmacopoeia Commission: Pharmacopoeia of the Peoples Republic of China. Chinese Medical Science and Technology Press 1: 573-575, 2010.
28. Lin JM, Wei LH, Chen YQ, Liu XX, Hong ZF, Sferra TJ and Peng J: Pien Tze Huang-induced apoptosis in human colon cancer HT-29 cells is associated with regulation of the Bcl-2 family and activation of caspase 3. Chin J Integr Med 17: 685-690, 2011.

29. Zhuang QC, Hong F, Shen AL, Zheng LP, Zeng JW, Lin W, Chen YQ, Sferra TJ, Hong ZF and Peng J: Pien Tze Huang inhibits tumor cell proliferation and promotes apoptosis via suppressing the STAT3 pathway in a colorectal cancer mouse model. Int J Oncol 40: 1569-1574, 2012.

30. Shen AL, Hong F, Liu LY, Lin JM, Zhuang QC, Hong ZF and Peng J: Effects of Pien Tze Huang on angiogenesis in vivo and in vitro. Chin J Integr Med 18: 431-436, 2012.

31. Shen AL, Hong F, Liu LY, Lin JM, Wei LH, Cai QY, Hong ZF and Peng J: Pien Tze Huang inhibits the proliferation of human colon carcinoma cells by arresting G1/S cell cycle progression. Oncol Lett 4: 767-770, 2012.

32. Shen AL, Chen YQ, Hong F, Lin JM, Wei LH, Hong ZF, Sferra TJ and Peng J: Pien Tze Huang suppresses IL-6-inducible STAT3 activation in human colon carcinoma cells through induction of SOCS3. Oncol Rep 28: 2125-2130, 2012.

33. Kaya M, Wada T, Akatsuka T, Kawaguchi S, Nagoya S, Shindoh M, Higashino F, Mezawa F, Okada F and Ishii S: Vascular endothelial growth factor expression in untreated osteosarcoma is predictive of pulmonary metastasis and poor prognosis. Clin Cancer Res 6: 572-577, 2000.

34. Maeda K, Chung YS, Ogawa Y, Takatsuka S, Kang SM, Ogawa M, Sawada T and Sowa M: Prognostic value of vascular endothelial growth factor expression in gastric carcinoma. Cancer 77: 858-863, 1996

35. Ferrara N, Gerber HP and LeCouter J: The biology of VEGF and its receptors. Nat Med 9: 669-676, 2003.

36. Ishigami SI, Arii S, Furutani M, Niwano M, Harada T, Mizumoto M, Mori A, Onodera $\mathrm{H}$ and Imamura M: Predictive value of vascular endothelial grow th factor (VEGF) in metastasis and prognosis of human colorectal cancer. Br J Cancer 78: 1379-1384, 1998.

37. Gille H, Kowalski J, Li B, LeCouter J, Moffat B, Zioncheck TF, Pelletier N and Ferrara N: Analysis of biological effects and signaling properties of Flt-1 (VEGFR-1) and KDR (VEGFR-2). A reassessment using novel receptor-specific vascular endothelial growth factor mutants. J Biol Chem 276: 3222-3230, 2001.

38. Fukumura D, Gohongi T, Kadambi A, Izumi Y, Ang J, Yun CO, Buerk DG, Huang PL and Jain RK: Predominant role of endothelial nitric oxide synthase in vascular endothelial growth factor-induced angiogenesis and vascular permeability. Proc Natl Acad Sci USA 98: 2604-2609, 2001

39. Sessa WC: eNOS at a glance. J Cell Sci 117: 2427-2429, 2004.

40. Vakkala M, Kahlos K, Lakari E, Paakko P, Kinnula V and Soini Y: Inducible nitric oxide synthase expression, apoptosis, and angiogenesis in situ and invasive breast carcinomas. Clin Cancer Res 6: 2408-2416, 2000.

41. Aaltomaa SH, Lipponen PK and Kosma VM: Inducible nitric oxide synthase (iNOS) expression and its prognostic value in prostate cancer. Anticancer Res 21: 3101-3106, 2001. 